\title{
Genetic diversity of Actinobacteria inhabiting water and sponges of Lake Baikal
}

IIMNOLOGY FRESHWATER

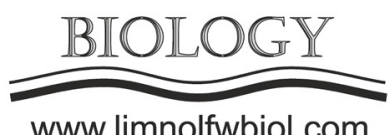

\author{
Lipko I.A. ${ }^{1 *}$, Krasnopeev A.Yu. ${ }^{1}$, Tikhonova I.V. ${ }^{1}$, Timoshkin O.A. ${ }^{1}$, Kabilov M.R. ${ }^{2}$, \\ Belykh O.I. ${ }^{1}$
}

\footnotetext{
${ }^{1}$ Limnological Institute, Siberian Branch of the Russian Academy of Sciences, Ulan-Batorskaya Str., 3, Irkutsk, 664033, Russia

${ }^{2}$ Institute of Chemical Biology and Fundamental Medicine, Siberian Branch of the Russian Academy of Sciences, Lavrentiev Ave., 8, Novosibirsk, 630090, Russia
}

\begin{abstract}
Actinobacteria is a ubiquitous major group in freshwater bacterioplankton and sponge microbial communities. We were first to analyse the diversity of Actinobacteria inhabiting Lake Baikal water and endemic sponges Lubomirskia baikalensis (healthy and diseased) using high - throughput $16 \mathrm{~S}$ rRNA sequencing. In total, we have assigned of three classes, fourteen orders and twenty-six families of Actinobacteria. The diversity and composition of actinobacteria were common in bacterioplankton and healthy sponges. The environmental crisis in the coastal zone of Lake Baikal led to a colonization of the L. baikalensis by benthic and soil-derived representatives of Actinobacteria. Some amplicon sequences of opportunistic genera were detected in the coastal water. The example of Actinobacteria has dramatically demonstrated the response of bacterial populations caused by negative environmental impact.
\end{abstract}

Keywords: freshwater sponge; actinobacteria; high-throughput 16S rRNA sequencing; Lake Baikal

\section{Introduction}

Phylum Actinobacteria is one of the largest taxonomic units in the domain Bacteria (Ventura et al., 2007). They represent a significant part of the microbial community in freshwater reservoirs (Garcia et al., 2014) and freshwater sponges (Gernet et al., 2005; Costa et al., 2013). In Lake Baikal, the phylum Actinobacteria is the prevalent component of the microbial communities in the water column and healthy sponges (Parfenova et al., 2013; Gladkikh et al., 2014; Seo et al., 2016; Kulakova et al., 2018). Negative ecological changes in the coastal zone of the lake, which began in 2010-2011, caused sickness and mass mortality of endemic sponges. The bleached and diseased L. baikalensis revealed a change in the composition and structure of the sponge microbial community, including Actinobacteria (Denikina et al., 2016; Kulakova et al., 2018;).

Through all studies, there are still no papers on the detailed composition of actinobacterial communities in baikalian sponges and bacterioplankton. We were aimed to reveal actinobacterial diversity in the lake water as well as healthy and diseased $L$. baikalensis and to analyse the actinobacteria composition before and during environmental crisis in the coastal zone of Lake Baikal.

\section{Materials and Methods}

We investigated 6 samples of diseased $L$. baikalensis (bleached, green with brown patches and maroon-violet biofouling) and 6 water samples collected in the local bays Bolshiye Koty and Listvennichny of Lake Baikal in 2011-2015. Total DNA was extracted using the DNA-Sorb B kit according Gladkikh et al. (2014). DNA amplification was performed using 343F and 806R bacterial primers targeting V3 and V4 regions of the 16S rRNA gene. High - throughput sequencing was carried out on the Illumina platform in SB RAS Genomics Core Facility (Russia). Bioinformatics analysis also included already published data on amplicon sequencing analysis of healthy L. baikalensis sponges and water samples from Lake Baikal (Parfenova et al., 2013; Gladkikh et al., 2014). Quality filters, chimera removal and operational taxonomic units clustering (threshold 97\%) were performed using Mothur v.1.39.5 (http:// www.mothur.org). The sequences were aligned using SILVA database (v.132 release, https://www.arb-silva. de). All calculations were performed with HPC-cluster «Akademik V.M. Matrosov» (Irkutsk, Russia; http:// hpc.icc.ru). 


\section{Results and Discussion}

The phylum Actinobacteria dominated the microbial community of the water column, particularly in the coastal zone, and contained from $32 \%$ to $69 \%$ of all sequences. They constitute a significant part of the microbial community in healthy and diseased $L$. baikalensis. In healthy sponges, actinobacteria ranged from $3.5 \%$ to $14 \%$ (Gladkikh et al., 2014; Seo et al., 2016), but in diseased ones their fraction remained at the same level of up to $8.6 \%$, except for bleached specimens.

Totally, we have assigned of three classes (Actinobacteria, Acidimicrobiia, and Thermoleophilia), fourteen orders and twenty-six families. Among them, classes Actinobacteria and Acidimicrobiia were ubiquitous and dominant in all L. baikalensis and water samples, whereas Ilumatobacteraceae (CL500-29), Microtrichaceae, and Sporichthyaceae (hocI_clade) were the prevalent families. Results of bioinformatics and cluster analysis for V3-V4 showed that in the coastal zone of Lake Baikal actinobacteria diversity and composition were similar until 2014 since the major phylotypes were common for water and sponges.

Actinobacterial diversity has transformed significantly and increased in diseased sponges by 2015. Some phylotypes of CL500-29 and hgcI_clade groups were negligible or eliminated from the $L$. baikalensis microbial community. The new phylotypes of CL500-29, Microtrichaceae and Microbacteriaceae, unclassified Acidimicrobiia, genera Ilumatobacter and Iamia in the sponge microbial community have become the dominant groups, comprising from $60 \%$ to $90 \%$ of the actinobacterial sequences. For the first time, two rare genera Gaiella and Parviterribacter (Thermoleophilia) were identified in diseased $L$. baikalensis. The nearest homologues (98\%-99\% similarity) were highly similar to Actinobacteria from the benthic community and bottom sediments, soils, groundwater, wastewaters and activated sludge reactors, and from reservoirs with high anthropogenic load. The new settled actinobacterial groups in diseased L. baikalensis seem to have different metabolic activities: having various enzyme activities, taking part in the nitrogen and phosphorus cycles, in bioremediation from the pollution with polycyclic aromatic and aliphatic hydrocarbons, decomposition of polymers, carbohydrates and esters. Furthermore, Microbacteriaceae exhibit fungicidal activity, suppressing the pathogens of aquatic and terrestrial animals. These Actinobacteria are likely able to protect diseased sponges from additional infection with aquatic fungal pathogens.

Some sequences from the shallow and pelagic water of Lake Baikal were homologous with the cultured opportunistic genera Actinomyces, Corynebacterium, Lawsonella, Rothia, Mycobacterium, and Gardnerella. These actinobacteria had an anthropogenic origin and could be potentially dangerous for people with weakened immunity.

\section{Conclusion}

In summary, the negative changes in the coastal zone of Lake Baikal caused the appearance of benthic and soil-derived representatives in the microbial community of the endemic sponge L. baikalensis. We assume that Actinobacteri $a$ associated with anthropogenic activities that have settled in sponges are likely to contribute to the longer existence of diseased L. baikalensis, since they decompose decaying sponge bodies and biofouling and protect weakened sponges from the effects of pathogenic fungi. This study highlights the ecological role of actinobacteria not only in the water column and in sponges but also in the environment as a whole.

\section{Acknowledgements}

The authors would like to thank Irkutsk Supercomputer Center of SB RAS for providing access to HPC-cluster «Akademik V.M. Matrosov» (http://hpc. icc.ru). Our thanks to Igor V. Khanaev for sampling. The study was carried out within the framework of State Task No. 0345-2020-0003 (AAAA-A16-1161221100616). This work was supported by RFBR grant no. 18-54-05005_arm_a.

\section{References}

Costa R., Keller-Costa T., Newton C.M. et al. 2013. Evidence for selective bacterial community structuring in the freshwater sponge Ephydatia fluviatilis. Microbial Ecology 65: 232-244. DOI: 10.1007/s00248-012-0102-2

Denikina N.N., Dzyuba E.V., Bel'kova N.L. et al. 2016. The first case of disease of the sponge Lubomirskia baicalensis: investigation of its microbiome. Biology Bulletin 3: 315-322. DOI: 10.7868/S0002332916030024

Garcia S.L., McMahon K.D., Grossart H-P. et al. 2014. Successful enrichment of the ubiquitous freshwater acI Actinobacteria. Environmental Microbiology Reports 6: 21-27. DOI: 10.1111/1758-2229.12104

Gernet C., Glockner F.O., Krohne G. et al. 2005. Microbial diversity of the freshwater sponge Spongilla lacustris. Microbial Ecology 50: 206-212. DOI: 10.1007/s00248-004-0172-x

Gladkikh A.S., Kaluzhnaya O.V., Belykh O.I. et al. 2014. Analysis of bacterial communities of two Lake Baikal endemic sponge species. Microbiology 83: 787-797. DOI: 10.1134/ s002626171406006x

Parfenova V.V., Gladkikh A.S., Belykh O.I. 2013. Comparative analysis of biodiversity in the planktonic and biofilm bacterial communities in Lake Baikal. Microbiology 82: 94-105. DOI: 10.1134/S0026261713010128

Kulakova N.V., Sakirko M.V., Adelshin R.V. et al. 2018. Brown rot syndrome and changes in the bacterial community of the Baikal sponge Lubomirskia baicalensis. Microbial Ecology 75: 1024-1034. DOI: 10.1007/s00248-017-1097-5

Seo E.Y., Jung D., Belykh O.I. et al. 2016. Comparison of bacterial diversity and species composition in three endemic Baikalian sponges. Annales de Limnologies 52: 27-32. DOI: 10.1051/limn/2015035

Ventura M., Canchaya C., Tauch A. et al. 2007. Genomics of Actinobacteria: tracing the volutionary history of an ancient phylura. Microbiology and Molecular Biology Reviews 71: 495-548. DOI:10.1128/MMBR.00005-07 